\title{
Electrochemical behaviour of zinc layer anodes used for galvanic protection of steel in reinforced concrete
}

\author{
David Garcia $^{a}$, Stéphane Laurens ${ }^{\text {a* }}$, Stéphane Panin ${ }^{b}$ \\ a Université de Toulouse, UPS, INSA, LMDC, 135 avenue de Rangueil 31077 Toulouse Cedex 4, France \\ ${ }^{\mathrm{b}} \mathrm{R} 3 \mathrm{~S}, 17$ Chemin des Vignes, 09270 Mazères, France
}

Received: 21 September 2018 / Accepted: 18 December 2018 / Published online: 20 December 2018

(C) The Author(s) 2018. This article is published with open access and licensed under a Creative Commons Attribution 4.0 International License.

\begin{abstract}
Steel corrosion is the most common reason for the premature deterioration of reinforced concrete structures. Consequently, cathodic protection of steel in concrete has been substantially developed during the past two decades - in particular galvanic protection. This consists of generating a natural macrocell corrosion system in which a sacrificial metallic anode (typically zinc) is used to apply a cathodic polarization to the corroding steel reinforcements, in order to mitigate or annihilate the corrosion kinetics. Although the general principle of cathodic protection is not questionable, the global design process can be significantly improved by increasing our knowledge of the electrochemical behaviours of the different components of the protecting system. Regarding zinc anodes in concrete, the literature is very scarce. The time evolution of such systems is also not rigorously addressed, ageing effects are systematically ignored and zinc anodes are usually considered as non-polarizable and inert over time. In this paper, the polarization response of a zinc layer anode (ZLA) in concrete electrolyte and its evolution over time are studied. The results show a rapid evolution of the ZLA behaviour once the protecting system is connected to steel reinforcements. Moreover, the characterization of ZLA provides relevant electrochemical properties for the numerical design of galvanic protection systems.
\end{abstract}

Keywords: : Reinforced concrete; Steel corrosion; Cathodic protection; Zinc layer anode; Electrochemical behaviour

\section{Introduction}

Galvanic protection of steel in concrete consists of generating a natural macrocell corrosion system in which a sacrificial metal (such as zinc) is involved as an anode to apply a cathodic polarization to the corroding reinforcing bars and consequently mitigate or annihilate the corrosion kinetics. In return, the sacrificial metal undergoes anodic polarization. Electrons produced by its oxidation (Eq. 1) are provided to the reinforcing steel through a metallic connection and then consumed by oxygen reduction at the steel-concrete interface (Eq. 2).

$$
\begin{aligned}
& \mathrm{Zn} \rightarrow \mathrm{Zn}^{2+}+2 e^{-} \\
& \frac{1}{2} \mathrm{O}_{2}+\mathrm{H}_{2} \mathrm{O}+2 e^{-} \rightarrow 2 \mathrm{OH}^{-}
\end{aligned}
$$

Given the separated spatial locations of the anodic and cathodic regions in the global electrochemical system, sacrificial cathodic protection has to be regarded as a macrocell corrosion system. Macrocell corrosion is characterized by a non-uniform potential field, an ionic current flowing in the concrete volume from the zinc anode to the reinforcing steel, and an electronic current exchanged through a metallic network.

In corroding reinforced concrete structures, the pre-existing macrocell corrosion current between active and passive steel areas will, at least, be mitigated by the cathodic polarization induced by the zinc anode and may even be annihilated if the protection system is properly designed.

Due to the potential gradient between the zinc anode and the reinforcing steel bars, a macrocell current is also generated in the concrete volume, corresponding to the so-called galvanic protection current. This protection current is therefore controlled by the electrical resistivity of the concrete. Abundant literature can be found regarding the experimental illustration of the mutual polarization between sacrificial anodes and the reinforcing steel to be protected [1-4], where the authors compare the performances of several galvanic anodes and developed protocols for laboratory and on-site current monitoring.

Zinc anodes in a concrete environment are always assumed to be non-polarizable and stable over time. Thus, for numerical simulations of these protection systems, a zinc

* Corresponding author: Stephane Laurens, E-mail: stephane.laurens@insa-toulouse.fr 
anode is modelled as having a constant potential $[4,5]$. This assumption is encouraged by the fact that zinc anodes in concrete have a polarization response that is very much steeper than that of reinforcing steel (active and/or passive). Therefore, zinc anodes are assumed to be very weakly polarized, if at all, by the electrical connection with the reinforcing steel in concrete. However, the corrosion of zinc anodes in concrete is an electrochemical phenomenon in which zinc cations are produced by oxidation and may interact with anionic ions, in the pore solution of the cementitious matrix, for example. Reaction products may settle at the zinc-concrete interface if these complexes have limited solubility, and a passivating film may be formed, limiting the zinc anodic reaction and inducing a drop in the global macrocell protection current.

The main alkaline component of concrete pore solution is calcium hydroxide, $\mathrm{Ca}(\mathrm{OH})_{2}$. This species leads to zinc passivation in concrete media by forming calcium hydroxyzincate as the main stable product (Eq. 3 and Eq. 4). Therefore, zinc layer anodes are always backed with activation paste to dissolve the passive film and theoretically keep the anode stable over time. Several authors have worked on the nature and stability of zinc oxides in different environments [7-10].

$$
\begin{aligned}
& \mathrm{Zn}^{2+}+2 \mathrm{OH}^{-} \rightarrow \mathrm{Zn}(\mathrm{OH})_{2} \\
& \mathrm{Ca}(\mathrm{OH})_{2}+2 \mathrm{Zn}(\mathrm{OH})_{2}+2 \mathrm{H}_{2} \mathrm{O} \\
& \rightarrow \mathrm{Ca}\left[\mathrm{Zn}(\mathrm{OH})_{3}\right]_{2} \cdot 2 \mathrm{H}_{2} \mathrm{O}
\end{aligned}
$$

The main purpose of this paper is not to determine the nature of zinc oxides but simply the influence of natural ageing of a zinc-based anodic system on the cathodic protection current it can supply. Actually, the literature regarding the electrochemical behaviour of zinc anodes in concrete and the possible ageing effects is very scarce. The short discussion above highlights the need to improve electrochemical knowledge of such anodic systems in concrete. This paper focuses on a specific zinc-based anodic system: the zinc layer anode (ZLA). The polarization behaviour of zinc layer anodes stuck on concrete is measured by means of potentiostatic tests carried out on small concrete slabs. The electrochemical properties achieved may be useful for the numerical design of galvanic cathodic protection implemented in RC structures.

\section{Experimental details}

A polarization curve expresses the steady-state behaviour of an electrochemical system under an external solicitation. It was experimentally assessed here by potentiostatic tests. The steady-state condition was ensured by following the experimental protocol proposed by Laurens et al. [11]. Potentiostatic tests were performed by using a measuring circuit having the following three electrodes:

- the working electrode: zinc layer;

- the counter electrode: titanium mesh;

- the reference electrode: saturated calomel electrode.
If the electrochemical behaviour of a uniform metallic corrosion system is controlled by charge transfer, ionic concentrations at the metal surface are equal to the bulk concentration and the polarization behaviour may be modelled by the usual Butler-Volmer equation [11-14] as follows (Eq. 5):

$$
\begin{aligned}
i=i_{\text {corr }}\left(\exp \left(\frac{2.3 \times\left(E-E_{\text {corr }}\right)}{\beta_{a}}\right)\right. & \\
& \left.-\exp \left(-\frac{2.3 \times\left(E_{\text {corr }}-E\right)}{\beta_{c}}\right)\right)
\end{aligned}
$$

where:

- $i\left(A / m^{2}\right)$ is the net current density flowing through the metal-electrolyte interface of the uniform system polarized at potential $E(V)$;

- $i_{\text {corr }}\left(\mathrm{A} / \mathrm{m}^{2}\right)$ is the corrosion current density at the free corrosion potential $E_{\text {corr }}(V)$;

- $\beta_{a}$ and $\beta_{c}(V /$ dec $)$ are the anodic and cathodic Tafel slopes, respectively, of the corrosion system.

The Butler-Volmer model is actually built as the algebraic sum of the current densities associated with anodic (metal dissolution) and cathodic (oxygen reduction) reactions. The net current density $i$ and the electrode potential $E$ are the state variables of the system while the free corrosion potential $E_{c o r r}$, the corrosion current density $i_{c o r r}$, and the anodic and cathodic Tafel slopes are electrochemical properties.

The experiments were carried out on 9 ZLA-coated concrete slabs with dimensions of $16 \times 16 \times 5 \mathrm{~cm}^{3}$ made from a single C35 concrete mix (Fig. 1 and Fig. 2). The mix proportions were as follows: a water-to-cement ratio of 0.6 , an aggregate-tocement ratio of 2.8 and a sand-to-cement ratio of 2.0. All specimens were demoulded 24 hours after pouring, and then cured for 7 days in a water tank at the controlled temperature of $20^{\circ} \mathrm{C} \pm 2{ }^{\circ} \mathrm{C}$. Before coating one face of the concrete slabs with a ZLA sheet, the dust and laitance at the surface were removed with a wire brush.

The zinc layer anodes were manufactured by corrPRE ${ }^{\circ}$ and were composed of a $250-\mu \mathrm{m}$-thick zinc sheet coated with a $900 \mu \mathrm{m}$-thick ion-conductive adhesive gel. The gel was used to:

- stick the zinc sheet on to the concrete surface;

- ensure the electrolytic contact at the zinc-concrete interface.

Even though no specific information about the gel composition is provided by the ZLA manufacturer, it is likely that it also helps to prevent passivation of the zinc and thus to maintain the reactivity of the sacrificial anode.

For each concrete slab, a ZLA sheet was cut with the same dimensions as the largest face of the slab, i.e. $16 \times 16 \mathrm{~cm}^{2}$ and served as the working electrode in the electrochemical experiments. The ZLA plate also had a hole in its centre. Once the ZLA sheet had been placed on the concrete slab, a small plastic tube was stuck over the hole in order to create a reservoir filled with a conductive solution. A saturated calomel reference electrode was then positioned in the tube to obtain the ZLA potential in the concrete. This experimental process ensured stable potential monitoring. In addition, the 
reference electrode was thus close to the working electrode, which implied a negligible ohmic drop due to the electrical resistivity of concrete.
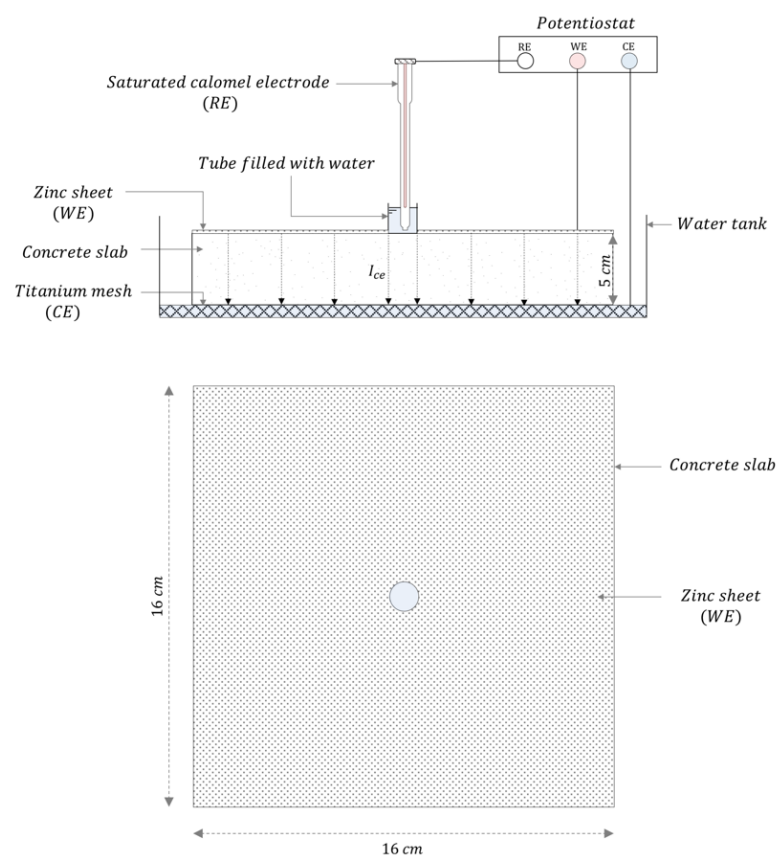

Figure 1. Description of the experimental setup.

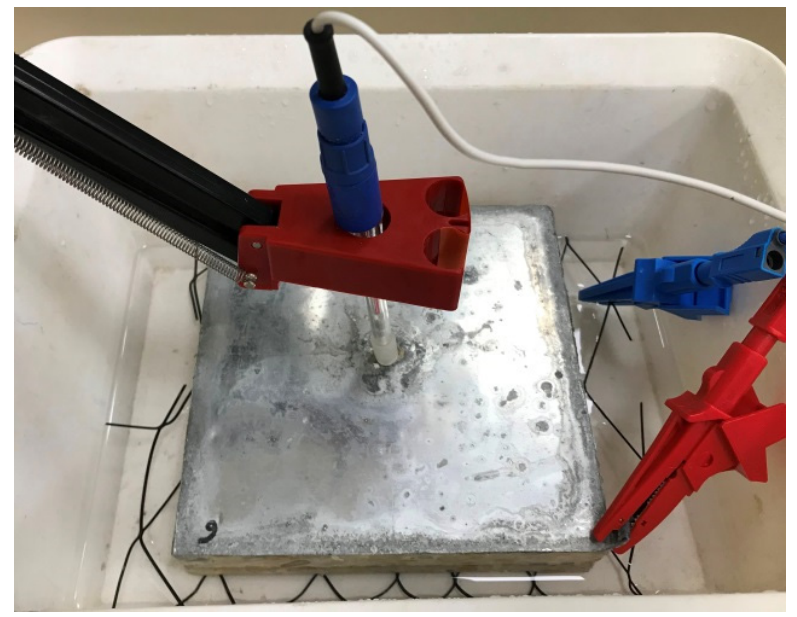

Figure 2. Photograph of the electrochemical cell.

The titanium mesh, used as a counter electrode, was flattened against the bottom of the concrete slab in a water tank. The concrete specimen was saturated but not fully immersed in water during the potentiostatic tests, in order to avoid any electrical artefacts. In fact, the water of the tank containing the counter electrode was only in contact with the bottom faces of the concrete slabs.

The geometry of the ZLA-coated concrete specimens was chosen to ensure a one-dimensional distribution of the polarizing current flowing within the concrete from the counter-electrode to the working electrode. Such a consideration is supported by the very small dimension of the hole with respect to the area of the ZLA sheet. Therefore, edge effects in the polarizing current distribution could be neglected at the centre of the slab, allowing the polarization of the ZLA sheet to be considered as uniform.

The polarization curve of the zinc layer anode was measured for three different ageing states:

- state 0 , corresponding to the initial state of ZLA, before any galvanic coupling with the reinforcing steel ( 9 specimens tested);

- state 1 , achieved after galvanic coupling for 10 days with external passive steel bars ( 9 specimens tested);

- state 2, achieved after galvanic coupling for 3 months with external passive steel bars ( 1 specimen tested).

The galvanic coupling of ZLA with passive steel bars allowed the natural ageing of the anode to be reproduced in realistic conditions (Fig. 3). A metallic wire electronically connected the zinc sheet stuck on the concrete slab specimen to the reinforcing passive steel embedded in a large concrete slab. The dimensions of the concrete slab were $50 \times 50 \times 7 \mathrm{~cm}^{3}$. Eight steel bars, $2 \mathrm{~cm}$ in diameter and $47 \mathrm{~cm}$ long, were used, with a concrete cover of $1.5 \mathrm{~cm}$. A water film was maintained between the small and the large concrete slabs by regular wetting, in order to facilitate their electrolytic connection. The output current was directly monitored over time by means of an ammeter (Fig. 4). This ageing process was applied to each of the 9 specimens for 10 days and the average galvanic current produced by the zinc sheets was about $5 \mathrm{~mA} / \mathrm{m}^{2}$ of the ZLA surface.

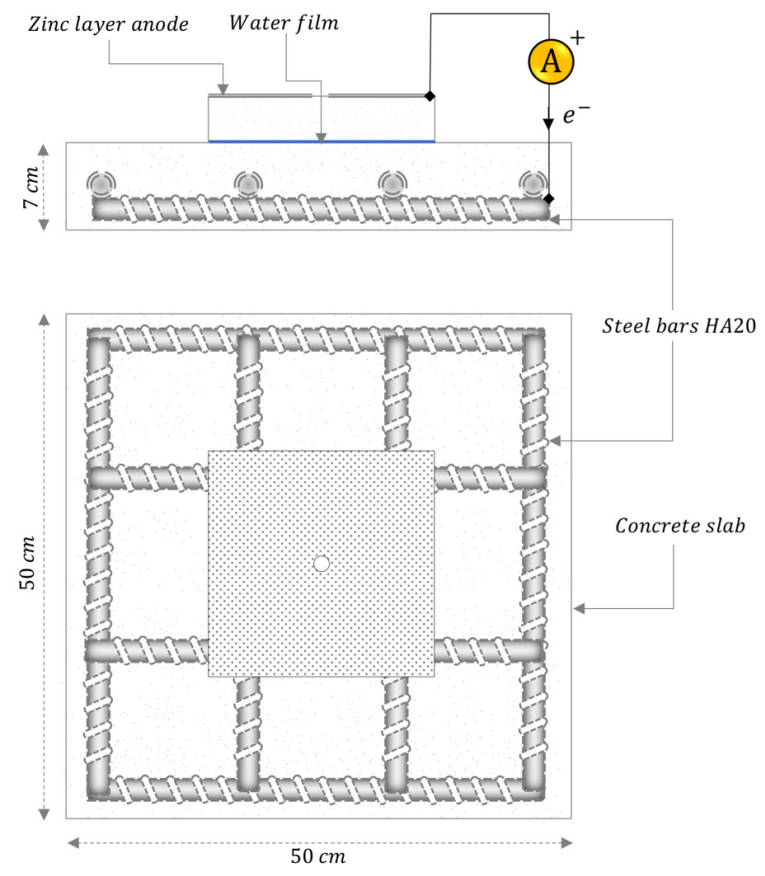

Figure 3. Experimental protocol for natural ageing of ZLA by galvanic coupling with an external passive steel layout.

The reversible potentiostatic step protocol applied to the ZLA stuck on the small concrete specimens is presented in Fig. 7. The electrochemical behaviour was investigated by applying polarizations ranging from $-15 \mathrm{mV}$ to $+15 \mathrm{mV}$ (Fig. 5). The duration of each step was set at $120 \mathrm{~s}$. It has to be mentioned that the maximum and minimum potentiostatic steps were 
not involved in the measurements since there were no associated reverse steps.

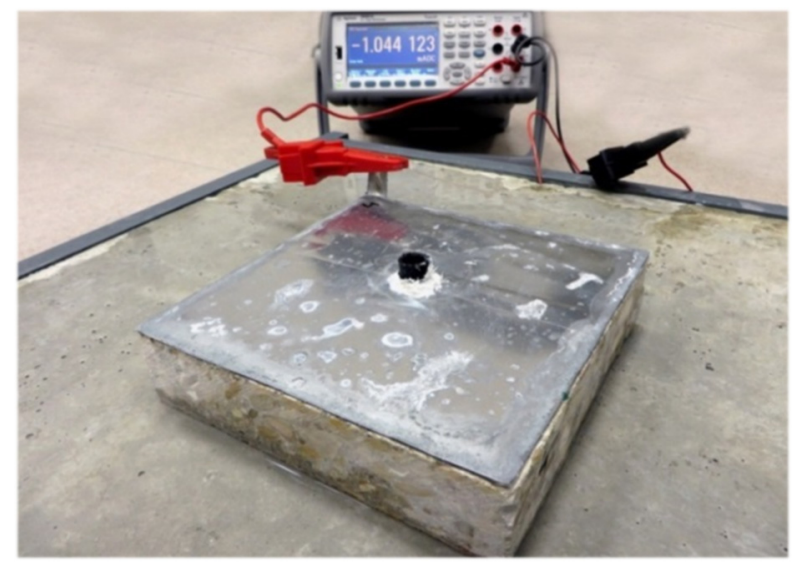

Figure 4. Photograph of the ZLA ageing experiment.

For each potentiostatic step, the time taken for the current response to stabilize could be very long. Therefore, the reversible potentiostatic step protocol was proposed to obtain a good approximation to asymptotic current values [11]. Fig. 6 displays the typical shape of the current response relative to anodic polarization. The response recorded the end of a particular potentiostatic ascending step $(\Delta E>0)$, referred to as $I_{\infty+1}$, overestimates the asymptotic value $I_{\infty}$. Conversely, in the reverse stage of the protocol, the current response at the end of the associated descending step $(\Delta E<$ 0 ), referred to as $I_{\infty_{-}}$, provides an underestimation of the asymptotic response. The asymptotic current value is thus well approximated by the average of $I_{\infty+}$ and $I_{\infty_{-}}$. In consequence, this experimental protocol ensured that polarization curves were achieved in real steady-state conditions.

As explained above, since the reference electrode was located immediately next to the ZLA sheet in the conductive solution reservoir, the potential measurements were not affected by any ohmic effects, i.e. an ohmic drop correction is not applicable here.

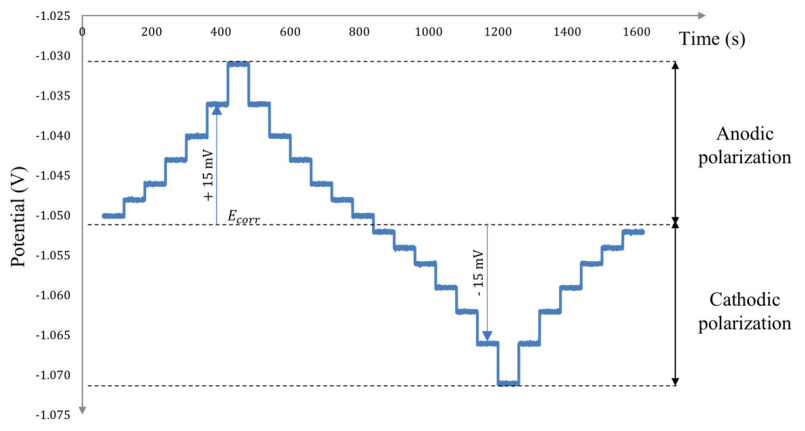

Figure 5. Potentiostatic polarization steps applied to the working electrode (ZLA).

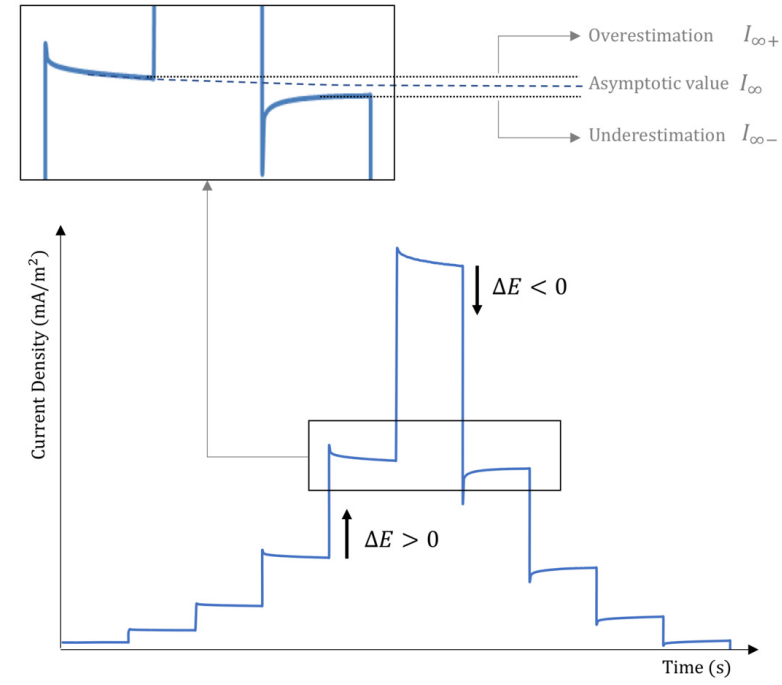

Figure 6. Illustration of the reversible potentiostatic step protocol.

\section{Experimental results and discussion}

Fig. 7 plots the experimental polarization results relative to the 9 ZLA specimens for state 0 , state 1 and state 2 . A polarization curve is built according to Eq. 5 by plotting the net current density $(i)$ produced at the zinc-concrete interface as a function of the over-potential $\left(E-E_{\text {corr }}\right)$ applied with respect to the free potential of zinc sheet in concrete $\left(E_{\text {corr }}\right)$. The following discussion pays particular attention to the anodic behaviour of the ZLA, which is involved in the galvanic protection of steel in reinforced concrete.

The first observation of the experimental data reveals a great difference in electrochemical behaviour between state 0 on the one hand, and states 1 and 2 on the other hand. Experimental points relative to state 2 (3 months' ageing process) are very close to the experimental curve obtained for state 1 (10 days' ageing process). This result indicates that no significant evolution of the anodic system behaviour occurred between 10 days and 3 months of ageing. However, a very short-term ZLA evolution is clearly observed between state 0 (initial state) and state 1 (10 days). Therefore, in the following discussion, state 1 is considered as a stabilized behaviour and state 2 is no longer discussed since the associated electrochemical behaviour was found to be equivalent to that of state 1.

Good homogeneity was achieved in the electrochemical responses of the 9 ZLA specimens for each ageing state ( 0 and 1). This correlation allowed a single Butler-Volmer polarization model to be fitted per ageing state. It can be deduced from these experimental results that the polarization level involved during the ageing process was very low, since the average current monitored between the ZLA sheets and the external passive steel network was only 5 $\mathrm{mA} / \mathrm{m}^{2}$ of zinc surface. On the experimental polarization diagrams (Fig. 7), this current level is associated with overpotentials of a few millivolts (about $+2 \mathrm{mV}$ at state 0 and +6 $\mathrm{mV}$ at state 1). This observation proves that the ZLA ageing protocol was only slightly aggressive. 
At stage 0 , very steep anodic and cathodic experimental responses were observed compared to the ones relative to stage 1 . In other words, for the same polarization level, the galvanic protection current provided by the ZLA at initial stage 0 was significantly higher than that supplied after the ageing process (state 1). For a polarization step of about $+12 \mathrm{mV}$, it can be observed from Fig. 7 that the ZLA provides about 7 times less protection current at state 1 than at state 0 $\left(70 \mathrm{~mA} / \mathrm{m}^{2}\right.$ at state 0 and $10 \mathrm{~mA} / \mathrm{m}^{2}$ at state 1$)$.

This result highlights a significantly higher activity of the sacrificial metal at stage 0 . Consequently, despite a slightly aggressive ageing protocol, the large decrease in the steepness of the ZLA anodic branch indicates a rapid evolution of the anode behaviour during the first few days after connection to the steel layout.

The observed evolution of the short-term behaviour of the ZLA could be explained by reactions between zinc oxides formed during the galvanic protection process and the constitutive ions of the adhesive gel. At this stage, it was not possible investigate these possible reactions further due to a lack of knowledge of the gel composition. Nevertheless, whatever the nature of these reactions, a relevant conclusion may be drawn regarding the on-site assessment of the galvanic system performance. The protection current measured immediately after electrical connection to the reinforcing steel does not reflect the long-term performance of the cathodic protection system. It appears necessary to wait for a few days before measuring the protection current supplied by the galvanic anodes. This leads to a more reliable assessment of the long-term efficiency of the cathodic protection system.

Fig. 8 presents the electrochemical properties identified by fitting the Butler-Volmer model on the experimental results for both ageing stages ( 0 and 1$)$. Bar charts with confidence intervals are used here to compare the 2 electrochemical states. The free corrosion potential $E_{\text {corr }}$ is found not to depend on the ageing process, which means that it appears as a stable property of ZLA over time. In contrast, the other three Butler-Volmer parameters are found to be very sensitive to ZLA ageing. A significant increase is seen for the Tafel slopes over time, reflecting the observed decrease in the polarization curve slope. On the other hand, the corrosion current density $i_{\text {corr }}$ is lowered by the ageing process.

As discussed above, the rapid time evolution of these electrochemical properties is likely to result from possible reactions between the ion-conductive gel components and the zinc sheet under anodic polarization. Moreover, both the increase in the anodic Tafel slope and the decrease in the corrosion current density reflect the amplification of the charge transfer resistance at the zinc-concrete interface, leading to a lowering of the ZLA ability to supply galvanic protection current.

Regarding the numerical design of ZLA galvanic protection systems, electrochemical properties relative to state 1 should be preferred for predicting the long-term performance. However, even though the ZLA behaviour appears to stabilize at state 1 , further studies are required to assess the very longterm ( $>3$ months) system efficiency.

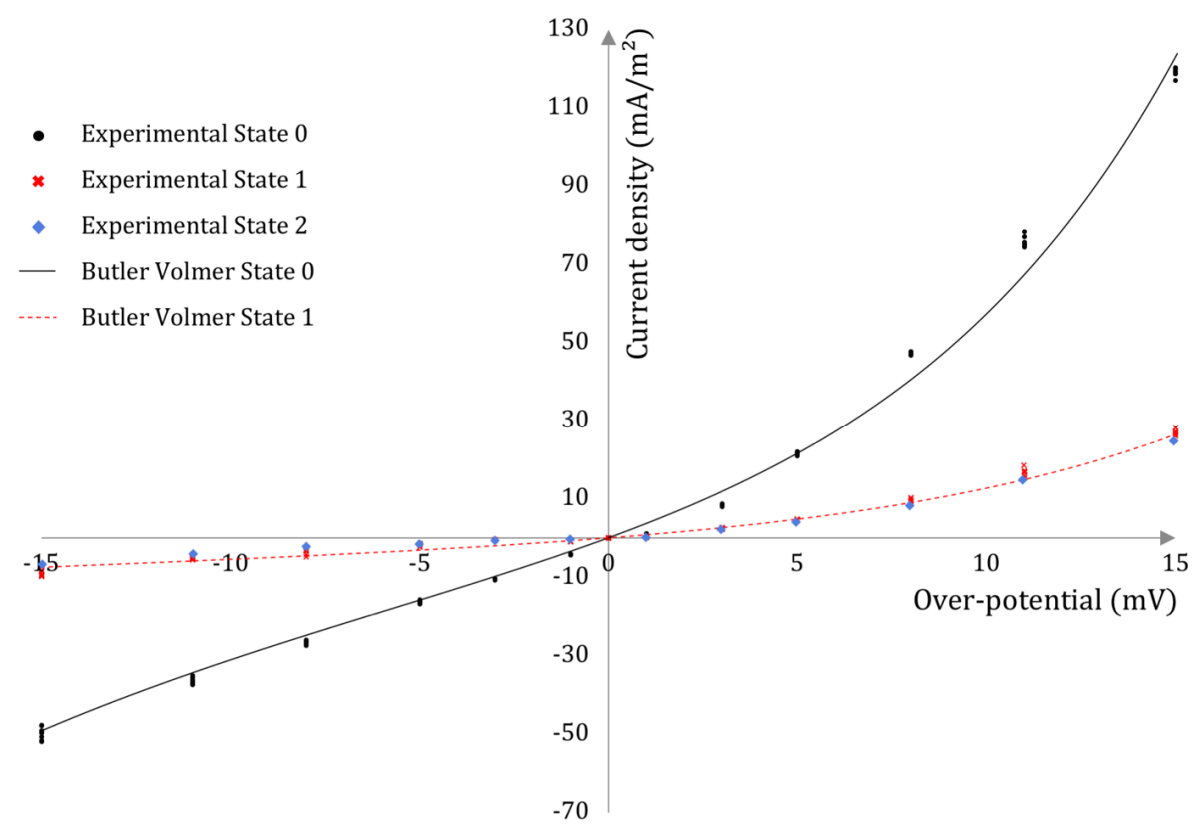

Figure 7. Polarization curves of zinc sheet for initial and aged states. 

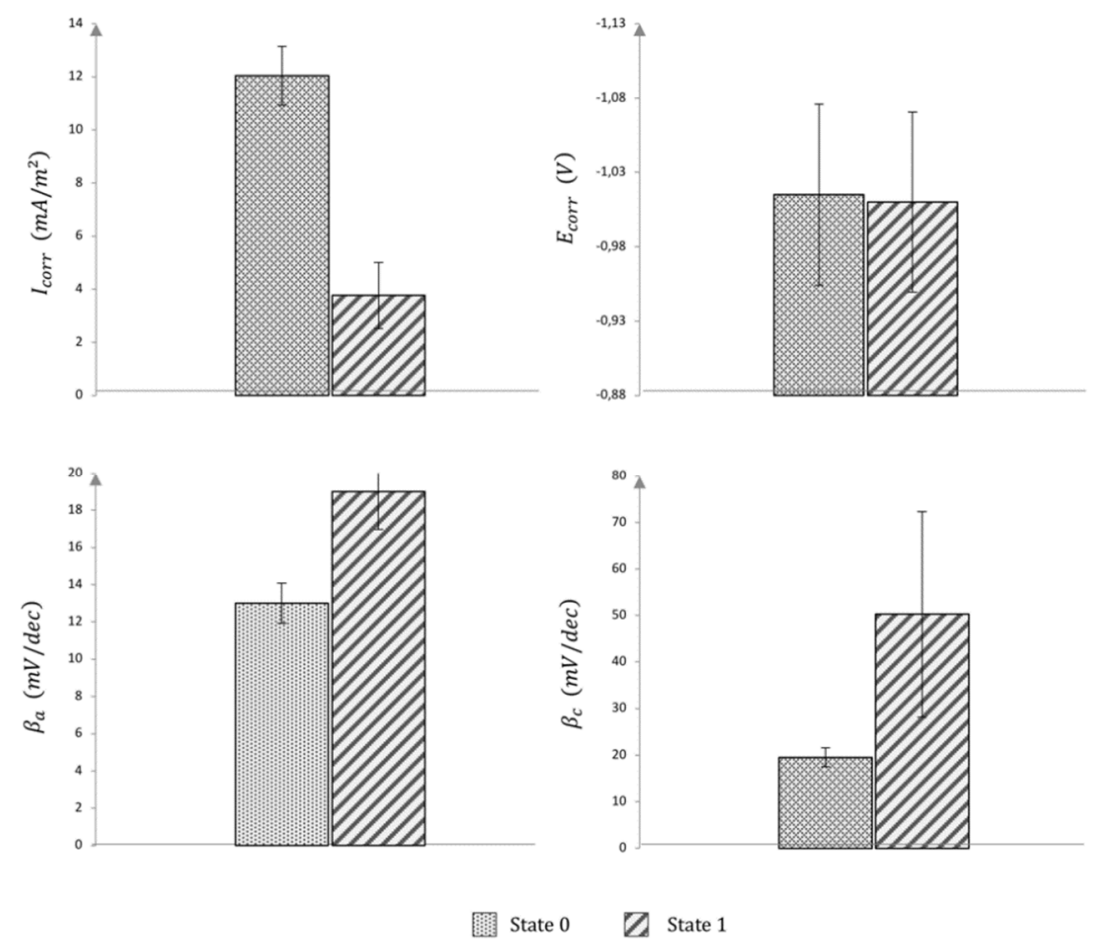

Figure 8. Bar chart with confidence intervals of the ZLA electrochemical properties identified for the 2 ageing states.

\section{Conclusion}

In the field of galvanic cathodic protection of steel in concrete, literature on the electrochemical behaviour and ageing effects of zinc anodes is very scarce. The zinc electrode is systematically considered as non-polarizable and inert over time. In this paper, the polarization response of a zinc layer anode (ZLA) in concrete electrolyte and its short-term time evolution are studied. The results actually show a rapid evolution of the ZLA behaviour once the protecting system is connected to steel reinforcements in concrete. This experimental observation proves that zinc layer anodes should not be considered as electrodes that are inert over time. Moreover, the output current measured immediately after connection to the reinforcing steel does not reflect the long-term behaviour of the protecting system. Consequently, it appears necessary to wait for a few days before measuring the protection current supplied by the galvanic anodes, so as to assess the long-term efficiency of the cathodic protection system more reliably. In order to achieve even more reliability, it appears necessary to rigorously address the longterm effects involving build-up of zinc-based corrosion products at the ZLA-concrete interface.

In the near future, it is likely that computer-aided design will be helpful to optimize cathodic protection systems in steel reinforced concrete. In this context, the results presented in this paper also help to extend the related electrochemical database as ZLA electrochemical properties are proposed from the fitting of the Butler-Volmer model on experimental polarization curves obtained at two different ageing states. These properties may be directly involved in the numerical design of cathodic protection systems based on zinc layer anodes.

\section{References}

[1] C. Christodoulou, C.I. Goodier, S.A. Austin, G.K. Glass, J. Webb, A new arrangement of galvanic anodes for the repair of reinforced concrete structures. Constr Build Mater (2014)50: 300-307.https://doi.org/10.1016/j.conbuildmat.2013.09.062

[2] S.P. Holmes, G.D. Wilcox, P.J. Robins, G.K. Glass, A.C. Roberts, Responsive behaviour of galvanic anodes in concrete and the basis for its utilisation. Corros Sci (2011) 53 https://doi.org/10.1016/j.corsci.2011.06.026

[3] M.D. Pritzl, H. Tabatabai, A. Ghorbanpoor, Laboratory Evaluation of Select Methods of Corrosion Prevention in Reinforced Concrete Bridges. Int J Concr Struct Mater (2014) 8: 201-212. https://doi.org/10.1007/s40069-014-0074-3

[4] G. Sergi, G., 2011. Ten-year results of galvanic sacrificial anodes in steel reinforced concrete. Materials and Corrosion 62, 98-104. https://doi.org/10.1002/maco.201005707

[5] L. Bertolini, E. Redaelli, Throwing power of cathodic prevention applied by means of sacrificial anodes to partially submerged marine reinforced concrete piles: Results of numerical simulations. Corros Sci (2009) 51: 2218-2230. https://doi.org/10.1016/j.corsci.2009.06.012

[6] K. Wang, P.R.L. Helene, P.J.M. Monteiro, Potential use of zinc in the repair of corroded reinforced concrete. Cem Concr Compos (2006) 28: 707-715. https://doi.org/10.1016/j.cemconcomp.2006.05.017

[7] A.-R. El-Sayed, H.S. Mohran, H.M. Abd El-Lateef, Inhibitive action of ferricyanide complex anion on both corrosion and passivation of zinc and zinc-nickel alloy in the alkaline solution. J Power Sources (2011) 196: 6573-6582. https://doi.org/10.1016/j.jpowsour.2011.03.057

[8] S.B. Farina, G.S. Duffó, Corrosion of zinc in simulated carbonated concrete pore solutions. Electrochimica Acta (2007) 52: 5131-5139. https://doi.org/10.1016/j.electacta.2007.01.014

[9] S.R. Yeomans, Chapter 1 - Galvanized Steel in Concrete: An Overview, in: Galvanized Steel Reinforcement in Concrete. Elsevier Science, Amsterdam, 2004, 1-289. https://doi.org/10.1016/B978-008044511-3/50016-5

[10] T.H. Muster, I.S. Cole, The protective nature of passivation films on zinc: surface charge. Corros Sci (2004) 46: 2319-2335. https://doi.org/10.1016/i.corsci.2004.01.002 
[11] S. Laurens, P. Hénocq, N. Rouleau, F. Deby, E. Samson, J. Marchand, B. Bissonnette, Steady-state polarization response of chloride-induced macrocell corrosion systems in steel reinforced concrete - numerical and experimental investigations. Cem Concr Res (2016) 79: 272-290.

https://doi.org/10.1016/j.cemconres.2015.09.021

[12] J. Warkus, M. Raupach, Modelling of reinforcement corrosion - Corrosion with extensive cathodes. Mater Corros (2006) 57: 920-925. https://doi.org/10.1002/maco.200604032

[13] J. Warkus, M. Raupach, J. Gulikers, Numerical modelling of corrosion - Theoretical backgrounds. Mater Corros (2006) 57: 614-617. https://doi.org/10.1002/maco.200603992

[14] J. Gulikers, M. Raupach, Numerical models for the propagation period of reinforcement corrosion - Comparison of a case study calculated by different researchers. Mater Corros (2006) 57: 618-627.

https://doi.org/10.1002/maco.200603993 